\title{
Low level maternal smoking and infant birthweight reduction: genetic contributions of GSTT1 and GSTM1 polymorphisms
}

\author{
Asta Danileviciute ${ }^{1}$, Regina Grazuleviciene ${ }^{1 *}$, Algimantas Paulauskas ${ }^{1}$, Ruta Nadisauskiene ${ }^{2}$ \\ and Mark J Nieuwenhuijsen ${ }^{3}$
}

\begin{abstract}
Background: Genetic susceptibility to tobacco smoke might modify the effect of smoking on pregnancy outcomes. Methods: We conducted a case-control study of 543 women who delivered singleton live births in Kaunas (Lithuania), examining the association between low-level tobacco smoke exposure (mean: 4.8 cigarettes/day) during pregnancy, GSTT1 and GSTM1 polymorphisms and birthweight of the infant. Multiple linear-regression analysis was performed adjusting for gestational age, maternal education, family status, body mass index, blood pressure, and parity. Subsequently, we tested for the interaction effect of maternal smoking, GSTT1 and GSTM1 genes polymorphisms with birthweight by adding all the product terms in the regression models.

Results: The findings suggested a birthweight reduction among light-smoking with the GSTT1-null genotype $(-162.9 \mathrm{~g}, P=0.041)$ and those with the GSTM1-null genotype $(-118.7 \mathrm{~g}, P=0.069)$. When a combination of these genotypes was considered, birthweight was significantly lower for infants of smoking women the carriers of the double-null genotypes $(-311.2 \mathrm{~g}, P=0.008)$. The interaction effect of maternal smoking, GSTM1 and GSTT1 genotypes was marginally significant on birthweight ( $-234.5 \mathrm{~g}, P=0.078)$. Among non-smokers, genotype did not independently confer an adverse effect on infant birthweight.

Conclusions: The study shows the GSTT1-null genotype, either presents only one or both with GSTM1-null genotype in a single subject, have a modifying effect on birthweight among smoking women even though their smoking is low level. Our data also indicate that identification of the group of susceptible subjects should be based on both environmental exposure and gene polymorphism. Findings of this study add additional evidence on the interplay among two key GST genes and maternal smoking on birth weight of newborns.
\end{abstract}

Keywords: Birthweight, GST polymorphisms, Smoking, Interaction

\section{Background}

Environmental factors contributing to reduced birthweight are a great concern because of the well-known relation of birthweight to infant mortality and adverse health effects in later life. Recent epidemiological studies have linked maternal tobacco-smoking and other environmental exposures to increased risk of low birth weight, preterm delivery, congenital anomalies, pregnancy loss, foetal growth, birthweight $[1,2]$. Investigators,

\footnotetext{
* Correspondence: r.grazuleviciene@gmf.vdu.lt

'Department of Environmental Sciences, Vytautas Magnus University, Donelaicio st. 58, 44248, Kaunas, Lithuania

Full list of author information is available at the end of the article
}

who have examined the issue, showed dose-response gradients in relation to the number of cigarettes smoked [3] and association between maternal smoking during pregnancy, impaired foetal growth and overweight in childhood [4]. Some studies reported an increased risks of low birth weight (LBW) and small for gestational age with heavier maternal smoking (> 10 cigarettes/day), as well as noting an increased risk for "very preterm" birth ( $<35$ weeks) [3]. It was reported that smoking even 20 cigarettes/day was not related to risk of preterm birth overall, but cotinine measured at the time of delivery was. A clear association and dose-response gradient was present for risk of foetal growth restriction [5] and 
birthweight reduction [6] and this effect was evident even in cases of a "minimal" consumption of 1-5 cigarettes per day. It was estimated that approximately $30 \%$ of growth-restricted neonates could be independently associated with maternal smoking [7].

Low birth weight of infant is recognised to be associated with demographic and environmental factors; however, tobacco-smoking remains the most important modifiable risk factor for foetal-growth restriction [8]. Maternal smoking during pregnancy, for heavy smokers, results in an approximately 150 - to 245 -g reduction in the average birthweight of infants $[3,9,10]$, however, it is generally accepted that there is no safe level of exposure to cigarette smoke [11]. In infants birthweight decreased as the mother's smoking increased, but the differences comparing 1-5 cig/day vs. 6-10 cig/day were not significant [6]. One of the explanations of tobacco smoking influence on infants birthweight is that individual genetic susceptibility to tobacco smoke might also have an effect on the foetal development [12]; - moreover, this variability may be related to the interplay of environmental exposures, such as exposure to cigarette smoke, and host-modified metabolic processes [13].

Several recent studies have represented the investigations how genetic susceptibility modulates risk of birthweight, infant birth size, small-for-gestational-age from environmental exposures such as cigarette smoke [13-16].

Numerous chemical compounds such as polycyclic aromatic hydrocarbons (PHA) in tobacco smoke are activated and detoxified by xenobiotic-metabolizing enzymes, such as glutathione S-transferase (GST) complex. Glutathione S-transferase M1 (GSTM1) is involved in the detoxification of a large number of xenobiotics $[17,18]$. The genetic polymorphism of GSTM1 may be a factor in determining the individual's susceptibility to the toxic effects of various xenobiotics, tobacco smoke being one among them. The deficiency of GSTM1 has been shown to impaired enzyme activities, increase DNA-adduct formation and cytogenic damage [14,19], and increase the susceptibility to both tobacco-smoke xenobiotics and small-for-gestational-age $[16,20]$. GSTM1 gene polymorphism is found to be present in 40 to $60 \%$ of most populations [17].

GSTT1-encoded enzymes catalyses the detoxification of xenobiotics, it is probable that GSTT1 plays a protective role against cell damage. The GSTT1-encoded enzymes are involved in the metabolism and detoxification of PAHs. It is possible that GST induction represents part of an adaptive response mechanism to chemical stress [20]. The frequency of the GSTT1-null allele has been reported to be 30 to $40 \%$ in Germany [21], whereas in a Swedish population, it is only $10 \%$ [22].

GSTM1 and GSTT1 null genes are the result of two homozygous deletions that result in a loss of functional activity [23]. Studies of the interaction between genes and toxic chemical exposure indicate that the GSTM1null and GSTT1-null genotypes have been associated with the effect of maternal smoking on duration of gestation, birth weight, and size $[13,14,16]$. Several recent studies have reported that genetic susceptibility modulates the risk of adverse pregnancy outcomes from tobacco smoke [15,16,24-31].

Different results have been presented by several authors [16]. In a case-control study, controlling for several confounding factors, the authors have shown that the maternal GSTT1-null genotype had a 1.6-fold reduced risk of infant-growth restriction. However, after adjustment for maternal smoking (categories less than 10 cigarettes per day and more than 10 cigarettes per day), the results are not statistically significant. There is evidence that the effect of cigarette-smoke exposure depends on the population characteristics: among the Japanese, the GSTM1-null genotype decreases foetal growth but this effect is not observed in Caucasians $[3,14]$.

Tobacco smoke is a complex mixture of numerous substances that include polycyclic aromatic hydrocarbons (PAHs) and N-nitrosamines among them. Recent studies have shown that there are significant associations between exposure to PAHs and reduced foetal growth and preterm birth [1]. Increasing PAH levels during pregnancy may increase the risk of foetal growth restriction [32], lung- function reduction in children, particularly for those whose mothers possessed the polymorphic CYP1A1*2A and GSTM1 deletion [26]. However, there is inconsistency in the relationship between GSTT1 and GSTM1 polymorphisms and smoking effects on foetal development. Variations in the CYP1A1 and GSTM1 genes that encode these enzymes could affect smoking behaviour by altering the levels and duration of tobacco-related PAHs and their metabolites in the body [33].

Although separate GSTM1 and GSTT1 gene deletions are well-studied functional variants, so far, there are only a limited numbers of studies published on the impacts of light cigarette smoking, GST metabolic gene polymorphism, and infant birth weight data.

In this study, we used a case-control design to examine the relationship between maternal smoking, GSTM1, GSTT1 polymorphism, and birth weight. We hypothesized those women with the GSTM1- and GSTT1- null genotype who are exposed to cigarette smoke during pregnancy are at elevated risk for newborn birthweight reduction.

\section{Methods}

\section{Participant and outcome assessment}

A prospective cohort study of pregnant women was conducted between 2007 and 2008 in Kaunas, Lithuania (Kaunas HiWATE cohort study). On their first visit to a general practitioner, all pregnant women living in Kaunas were invited to join the cohort and answer to the first 
questionnaire. We recruited these women for the prospective cohort study, enrolling them at 23-35 weeks of gestation at the four prenatal care clinics affiliated to the hospitals of the Kaunas University of Medicine. Participation was on a voluntary basis and the women were enrolled in the study only if they consented to participate in the cohort. The research protocol was approved by the Lithuanian Bioethics Committee and informed consent was obtained from all subjects. A special questionnaire was evolved to interview the women who agreed to participate in the genetic study and blood samples for genetic analysis was collected. Details of the methods and study subjects have been published elsewhere [34]. The subjects of this case-control study were 543 women, who delivered singleton live births at the four hospitals affiliated to the Lithuanian University of Health Science. Multiple births or newborns with major births defects were excluded.

Pregnant women of the cohort were asked to answer second questionnaire provided to them at the clinic before delivery. The interview contained a number of variables including demographics (age, education and family status); reproductive history (miscarriage); job characteristics; self-reported psychosocial stress; health behaviour; diseases; maternal smoking; paternal smoking. The selfreported stress of the respondents was assessed by the following thesis: "My daily activities are very trying and stressful". Four respondent options were used to define stress: this describes my state (1) very well, (2) fairly well, (3) not very well, (4) not at all. Values 1 and 2 were considered to represent stress; 3 and 4 represented no stress.

The women were followed up with regard to pregnancy outcomes by the research staff. Pregnancy outcomes were ascertained chiefly from computerised hospital admission files and by abstraction of medical records. In this study, infant birthweight was measured in the delivery room by a trained nurse and was accurate to $1 \mathrm{~g}$. The age of gestation was calculated using the data of birth as reported on the birth certificate and the 1st day of the last menstrual period as was ascertained at first interview, and by ultrasound examination.

\section{Smoking exposure}

Data regarding smoking behaviour were acquired through face-to-face interviewing. The trained research assistant in person in the hospital setting asked the women to report their daily cigarette consumption both before and during pregnancy. Woman had to answer the questions, "How many cigarettes did you smoke before pregnancy?" and "How many cigarettes did you smoke during pregnancy?" A mother was defined as smokers if she reported smoking at least one cigarette per day during pregnancy. In this study, data on mothers were categorised into two groups with respect to their cigarette smoking habits: those who did not smoke and those women who continue smoking during pregnancy. The parent was defined as a smoker if he smoked at least one cigarette per day. To assess smoking level we calculated the mean number cigarettes smoked per day.

\section{Genotyping}

The genomic DNA was extracted according to a standard protocol. The gene GSTM1- null (GenBank accession no. X68676) and GSTT1-null (GenBank accession no. AP000351) genotypes were identified by the multiplex polymerase chain reaction (PCR) in peripheral blood DNA samples. The details of this method for the detection of polymorphism of GSTT1 and GSTM1 can be found elsewhere [35]. This method allows the detection of the presence of the genotype (at least 1 allele present: AA or $\mathrm{Aa}$ ) or its absence (complete deletion of both alleles: aa).

Maternal blood samples were collected in vials containing EDTA and stored at a temperature of $-20^{\circ} \mathrm{C}$. DNA was purified from the peripheral blood using DNA purification kits (MBI "Fermentas", Vilnius, Lithuania). DNA concentrations were quantified with a spectrophotometer (Eppendorrf BioPhotometer, 61310488, Hamburg, Germany). A PCR-based study of GSTM1 and GSTT1 polymorphism was carried out according to the method described previously [24]. The research staffs were blinded to outcome. The primers used for PCR were as follows: GSTM1 forward5'-GAA CTC CCT GAA AAG CTA AAG C-3/and reverse $5^{\prime}$-GTT GGG CTC AAA TAT ACG GTG G-3'; GSTT1 forward 5'-TTC CTT ACT GGT CCT CAC ATC TC-3' and reverse 5'-TCA CCG GAT CAT GGC CAG CA-3'. As internal control, a 268-bp fragment of the human $\beta$-globin gene (GenBank accession no. U01317) was coamplified with a second set of primers (5'-CAA CTT CAT CCA CGT TCA CC-3') and (5'-GAA GAG CCA AGG ACA GGT AC- 3') (Biomers.net - the biopolymer factory, Germany). PCR was carried out in a final volume of $25 \mu \mathrm{l}$. The procedure followed for PCR was: primary denaturation at $94^{\circ} \mathrm{C}$ for $5 \mathrm{~min}$, denaturation at $94^{\circ} \mathrm{C}$ for $1 \mathrm{~min}$, annealing at $60^{\circ} \mathrm{C}$ for $1 \mathrm{~min}$, extension at $72^{\circ} \mathrm{C}$ for $1 \mathrm{~min}, 30$ cycles were conducted. Final extension was at $72^{\circ} \mathrm{C}$ for $10 \mathrm{~min}$. The PCR products were electrophoresed in $2 \%$ agarose gels and stained in ethidium bromide. The DNA bands were visualised by UV transillumination (EASY Win32, Herolab, Germany). GSTM1 and GSTT1 polymorphisms were coded as present (GSTM1-1 and GSTT1-1) or null (GSTM1-0 and GSTT1-0). To confirm the analyses we repeated genotyping for GSTM1 and for GSTT1 in 150 subjects. The genotyping consistency rates were $100 \%$ for both GSTM1 and GSTT1.

\section{Statistical methods}

We first examined the associations between the maternal characteristics and smoking status during pregnancy, 
in addition to the birthweight of the infants, by the Student's $t$-test. We then used multiple linear-regression models to estimate the association of maternal cigarette smoking during pregnancy and the maternal genetic polymorphism in relation to birthweight of the newborn, with adjustment for major covariates. These included prepregnancy body mass index $\left(\mathrm{BMI}=\right.$ weight $/$ height $\left.^{2}\right)$, blood pressure, parity, gestational age, education and family status. The Mantel - Haenszel test was used to test the interaction between GSTTI and GSTM1 on maternal smoking. Results from these association analyses were further verified using regression models to test the associations of GSTT1 GSTM1 and maternal smoking, including the effect from the interaction between the two genes. The subgroups were defined for maternal smoking status during pregnancy (no vs. yes) and genotypes for GSTT1 (null vs. present) and GSTM1 (null vs. present). We tested the gene-cigarette smoke interaction effect for birthweight reduction by adding all the product term (both 2-way and 3-way terms) in the model adjusting for potential effect modifiers. In the analyses, beta $(\beta)$ represents the difference in mean birthweight (continuous variable) for cigarette smoking between the variant genotype after adjustment for the selected effect modifiers. Statistical significance was defined as $P<0.05$. All statistical analyses were carried out using the SPSS software for Windows version 12.0.1.

\section{Results}

The analysis included 543 pregnant women: 460 nonsmokers and 83 continuous smokers during pregnancy. The mean number cigarettes smoked per day were 4.8. Before pregnancy smoked 140 (25.7\%) study subjects, among them $42.9 \%$ continuous smokers during pregnancy and $57.1 \%$ non-smokers during pregnancy. Prevalence of passive smoking at home (husband smoking) among continuous smokers during pregnancy was $91 \%$ and among non-smokers during pregnancy it was $50.4 \%$. A total of $95.9 \%$ women were Lithuanian and the 2 groups did not differ in ethnicity.

We also conducted analyses comparing questionnaire data and birth certificate data on various characteristics among participants and non-participants. The mean birthweight and gestational duration were similar among the two groups. These two groups did not differed by ethnic group, however, non-participating mothers were younger, less educated (did not graduate from university, $46.6 \%$ vs. $54.3 \%$ ), more often smokers (smokers, $9.6 \%$ vs. $6.9 \%$ ), and did have fewer prior births (no child, $64.1 \%$ vs. $45.1 \%$ ), than that of participants.

We found that among 543 participant, 450 (82.9\%) possessed at least one copy of the functional gene, GSTT1-plus genotype and the remaining 93 (17.1\%) had the GSTT1-null genotype. The GSTM1 gene among 543 study subjects, 293 (54\%) possessed at least one copy of the functional gene, GSTM1-plus genotype and the remaining 250 (46.0\%) had the GSTM1-null genotype. The carriers of the double-null genotypes comprised $8.7 \%$ of the total population studied. For the GSTM1 and GSTT1 polymorphisms, we were unable to determine whether they were in Hardy-Weinberg equilibrium because heterozygous individuals could not be distinguished from homozygous wild type.

Maternal characteristics with reference to the tobacco smoke exposure status are presented in Table 1. The nonexposed and exposed groups were similar in terms of the maternal prepregnancy BMI, hypertension, perceived stress, history of miscarriage, parity, sex of infant, and ethnic group, whereas the two groups differed with reference to maternal age, education and family status $(P<0.001)$. The mean birthweight of the infants was $3399.0 \mathrm{~g}$ for the nonexposed group and that for the exposed group was $3284.1 \mathrm{~g}$, but there was no statistically significant difference $(P=0.132)$. The mean gestational age was 38.9 weeks for the both groups. In terms of the frequency of the GSTM1-null genotype, women in the group exposed to tobacco smoke and the group nonexposed were similar $(41.0 \%$ and $47.0 \%, P=0.340)$, whereas the GSTT1-null genotype was found in $26.5 \%$ of the smokers and in $15.4 \%$ of the non-smokers $(P=0.018)$.

Table 2 presents the influence of maternal characteristics on the birthweight of the infants as the difference in mean birthweight in relation to the maternal characteristics listed in each row.

The characteristics that positively affected the crude mean birthweight were increased BMI, hypertension and gestational age. Low levels of education, not married status and previous preterm were associated with reduction in the mean birthweight. In terms of the GSTM1- and GSTT1- genotype frequencies, there was no significant influence on the crude birthweight of infants. After adjustment for the BMI, gestational age and loss outliers, the maternal characteristics that affected the reduction in birthweight were as follows: low education levels (-155.3 g), maternal smoking during pregnancy $(-137.0 \mathrm{~g})$, parental smoking (-93.3 g) and GSTT1-null genotype in smokers (-211.8 g, $P=0.036)$. When both GSTT1- and GSTM1null genotypes were considered, continuous maternal smoking during pregnancy was associated with a mean reduction of $340.4 \mathrm{~g}(P=0.016)$ in birthweight of infants.

Table 3 presents the crude and adjusted combined associations of continuous maternal smoking during pregnancy and maternal GSTT1 and GSTM1 genotypes with reference to infant birthweight, where $\beta$ represents the difference in mean birthweight between each subgroup and the reference group.

After complete adjustment for gestational age, BMI, education, family status, parity and hypertension, the 
Table 1 Characteristics of pregnant women and their newborn infants according to exposure to cigarette smoke during pregnancy

\section{Maternal characteristics}

continuous and binary

Maternal smoking status during pregnancy

\begin{tabular}{|c|c|c|c|}
\hline continuous and binary & Non-smoking $(n=460)$ & Smoking $(n=83)$ & $P$-value \\
\hline Maternal age, mean (SD), years & $29.0(5.0)$ & $26.4(5.7)$ & $<0.001$ \\
\hline Maternal height, mean (SD), cm & $167.6(5.7)$ & $167.3(7.2)$ & 0.703 \\
\hline Maternal weight, mean (SD), kg & $75.3(12.5)$ & $77.4(15.2)$ & 0.250 \\
\hline BMl, mean (SD) & $26.8(4.3)$ & $27.6(4.8)$ & 0.136 \\
\hline Gestational age, mean (SD), weeks & $38.9(2.2)$ & $38.9(2.2)$ & 0.979 \\
\hline Birthweight, mean (SD), g & $3399.0(639.2)$ & $3284.1(632.5)$ & 0.132 \\
\hline Birth length, mean (SD), cm & $51.3(3.1)$ & $50.8(3.5)$ & 0.150 \\
\hline \multicolumn{4}{|l|}{ Infant sex, n (\%) } \\
\hline male & $245(53.3)$ & $47(56.6)$ & 0.737 \\
\hline female & $213(46.3)$ & $36(43.4)$ & \\
\hline \multicolumn{4}{|l|}{ Education, n (\%) } \\
\hline university, college & $437(95.0)$ & $53(63.9)$ & $<0.001$ \\
\hline$\leq 12$ years & $23(5.0)$ & $30(36.1)$ & \\
\hline \multicolumn{4}{|l|}{ Marital status, n (\%) } \\
\hline married & $377(82.0)$ & $40(48.2)$ & $<0.001$ \\
\hline not married & $83(18.0)$ & $43(51.8)$ & \\
\hline \multicolumn{4}{|l|}{ Parity, n (\%) } \\
\hline $1^{\text {st }}$ & $217(47.2)$ & $38(45.8)$ & 0.905 \\
\hline $2^{\text {nd }}$ and more & $243(52.8)$ & 45 (54.2) & \\
\hline \multicolumn{4}{|l|}{ Miscarriage, n (\%) } \\
\hline no prior & $369(80.2)$ & $70(84.3)$ & 0.450 \\
\hline yes & $91(19.8)$ & $13(15.7)$ & \\
\hline \multicolumn{4}{|l|}{ Blood pressure, n (\%) } \\
\hline$<120 / 80 \mathrm{~mm} / \mathrm{Hg}$ & $324(70.4)$ & $56(67.5)$ & 0.604 \\
\hline$>120 / 80 \mathrm{~mm} / \mathrm{Hg}$ & 136 (29.6) & $27(32.5)$ & \\
\hline \multicolumn{4}{|l|}{ Stress, n (\%) } \\
\hline no & $379(82.4)$ & $64(77.1)$ & 0.281 \\
\hline yes & 81 (17.6) & $19(22.9)$ & \\
\hline \multicolumn{4}{|l|}{ Ethnic group, n (\%) } \\
\hline Lithuanian & 441 (95.9) & $80(96.4)$ & 0.571 \\
\hline other & $19(4.1)$ & $3(3.6)$ & \\
\hline \multicolumn{4}{|l|}{ Passive smoking } \\
\hline Yes & $235(50.4)$ & 75 (91.0) & $<0.001$ \\
\hline No & $225(49.6)$ & $8(9.0)$ & \\
\hline \multicolumn{4}{|l|}{ GSTT1, n (\%) } \\
\hline present & $389(84.6)$ & $61(73.5)$ & 0.018 \\
\hline null & $71(15.4)$ & $22(26.5)$ & \\
\hline \multicolumn{4}{|l|}{ GSTM1, n (\%) } \\
\hline present & $244(53.0)$ & $49(59.0)$ & 0.340 \\
\hline null & $216(47.0)$ & $34(41.0)$ & \\
\hline
\end{tabular}

SD standard deviation of the variability of individual observations. 
Table 2 Influence of maternal characteristics on infant's birthweight assessed by the crude and adjusted coefficient $B$ in linear regression $\left(n=539^{\dagger}\right)$

\begin{tabular}{|c|c|c|c|c|c|c|}
\hline Maternal characteristics continuous and binary & B crude & SE & $P$-value & $B^{*}$ adjusted & SE & $P$-value \\
\hline$\overline{\mathrm{BMI}^{* *}}$ & 42.7 & 6.0 & $<0.001$ & & & \\
\hline Gestational age, week $^{* *}$ & 191.0 & 9.2 & $<0.001$ & & & \\
\hline Age $<20$ or $>30$ years & -34.2 & 56.7 & 0.547 & -11.43 & 41.3 & 0.783 \\
\hline Low education, $\leq 12$ years & -279.7 & 91.7 & 0.002 & -155.3 & 67.2 & 0.021 \\
\hline Not married & -148.9 & 64.7 & 0.022 & -87.1 & 47.2 & 0.066 \\
\hline Parity 2nd and more & 49.5 & 55.0 & 0.368 & 90.8 & 39.9 & 0.023 \\
\hline Miscarriage & 15.2 & 69.7 & 0.828 & 4.0 & 50.8 & 0.937 \\
\hline Previous preterm & -400.1 & 135.2 & 0.003 & 82.5 & 100.0 & 0.409 \\
\hline Maternal stress & -94.7 & 70.7 & 0.181 & 5.4 & 51.6 & 0.916 \\
\hline Blood pressure $>120 / 80 \mathrm{~mm} / \mathrm{Hg}$ & 189.4 & 76.6 & 0.014 & 87.5 & 59.0 & 0.139 \\
\hline Maternal smoking & -114.9 & 76.1 & 0.132 & -137.0 & 55.2 & 0.013 \\
\hline Parental smoking & -86.9 & 55.0 & 0.115 & -93.3 & 40.1 & 0.020 \\
\hline GSTT1 null vs present & -104.1 & 72.7 & 0.153 & -72.7 & 52.7 & 0.170 \\
\hline GSTM1 null vs present & -25.3 & 55.1 & 0.646 & -26.0 & 40.0 & 0.516 \\
\hline GSTT1 null \& smoking & -207.0 & 138.9 & 0.137 & -211.8 & 100.8 & 0.036 \\
\hline GSTM1 null \& smoking & -60.4 & 113.3 & 0.594 & -150.2 & 82.4 & 0.069 \\
\hline GSTT1 \& GSTM1 null and smoking & -294.0 & 194.4 & 0.131 & -340.4 & 141.2 & 0.016 \\
\hline
\end{tabular}

B represent the difference in mean birthweight for maternal characteristics in each row.

$B^{*}$ adjusted for body mass index, gestational age and loss outlier.

${ }^{* *}$ Continuous variable.

SE standard error of the difference between the means.

${ }^{+}$Excluded outliers.

reduction in birthweight (analysed as a continuous variable) for continuous smokers was $83.4 \mathrm{~g}(P=0.073)$. Among non-smoking mothers, the GSTT1-null genotype alone did not confer a significant adverse effect on birthweight $(-22.6 \mathrm{~g}, P=0.345)$. The findings suggested a birthweight reduction among low-level smoking mothers with the GSTT1-null genotype $(-162.9 \mathrm{~g}, P=0.041)$. Maternal smoking was associated with a mean reduction of $58.8 \mathrm{~g}$ in birthweight for the GSTM1-plus and $118.7 \mathrm{~g}, P=0.069$ for the GSTM1-null genotypes; nevertheless, there was no statistically significant difference. When a combination of these genotypes was considered, a modifying effect was revealed and birthweight was significantly lower for infants of smoking women carriers of the double-null genotypes $(-311.2 \mathrm{~g} ; P=0.008)$. The interaction effect of maternal smoking, GSTM1 and GSTT1 genotypes was marginally significant on birthweight $(-234.5 \mathrm{~g} ; P=0.078)$.

\section{Discussion}

In this molecular epidemiological study on maternal cigarette smoking and genetic determinants of xenobiotic metabolism, we found some evidence that the effects of maternal smoking on infant birthweight were modified by the maternal GSTT1 and GSTM1 genotypes. This study shows that even light maternal smoking (mean: 4.8 cigarettes/day) has an increased risk for infant birthweight reduction among genetically susceptible woman. Smokers with the variant GSTT1-null genotype had babies with lower mean birthweight $(162.9 \mathrm{~g})$ than non-smokers with the same genotype $(\mathrm{P}=0.041)$, while smokers with the variant GSTM1-null genotype had babies with lower mean birthweight $(118.7 \mathrm{~g})$ than nonsmokers with the same genotype $(P=0.069)$. We also found a gene-gene interaction among smokers. A combination of the GSTM1-null and the GSTT1-null genotypes has been found to exacerbate the effect of maternal exposure to tobacco-smoking on the birthweight of infants more than the presence of either genotype alone: $-311.2 \mathrm{~g}$, $\mathrm{P}=0.008$ in smokers vs. $10.1 \mathrm{~g}, \mathrm{P}=0.447$ in non-smokers. An interaction effect of maternal smoking, GSTM1 and GSTT1 genotypes was marginally significant on birthweight $(-234.5 \mathrm{~g}, P=0.078)$. All associations were assessed after a number of relevant covariates were statistically controlled. These data and previous studies reported findings [18] suggest that the observed reductions in infants' birthweight from this sample could be related to the main effects of prenatal exposure to tobacco.

Consistent with earlier studies, we found that maternal cigarette-smoking reduced the birthweight of infants $[8,36]$ and that infant birthweight may vary in relation to gestational age, BMI, parity, and other variables of the population considered in the corresponding study $[5,37]$. 
Table 3 Associations between maternal smoking during pregnancy and infant birthweight by maternal GSTT1

and GSTM1 genotype assessed by the crude and adjusted coefficient $B$ in linear regression

\begin{tabular}{|c|c|c|c|c|c|}
\hline Genotype & Smoking status during pregnancy & Birthweight, g & Birthweight, $g \beta^{+}$crude (SE) $P$ & Birthweight, $g \beta^{++}$adjusted (SE) P & Birthweight, g $\beta^{++}$adjusted (SE) $P$ \\
\hline \multirow[t]{2}{*}{ Total sample } & Non-smoking $(n=456)$ & 3390.3 & Referent & Referent & Referent \\
\hline & Smoking $(n=83)$ & 3284.1 & $-86.5(57.5) 0.066$ & $-83.4(57.1) 0.073$ & $-83.4(57.1) 0.073$ \\
\hline \multicolumn{6}{|l|}{ GSTT1 } \\
\hline Present & Non-smoking $(n=385)$ & 3401.4 & Referent & Referent & Referent \\
\hline Present & Smoking $(n=61)$ & 3320.7 & $-70.4(65.7) 0.143$ & $-72.3(65.1) 0.134$ & $-38.8(57.6) 0.250$ \\
\hline$\overline{\text { Null }}$ & Non-smoking $(n=71)$ & 3330.0 & Referent & Referent & $-22.6(57.6) 0.345$ \\
\hline Null & Smoking $(n=22)$ & 3182.9 & $-115.9(129) 0.186$ & $-123.7(131) 0.175$ & $-162.9(93.0) 0.041$ \\
\hline \multicolumn{3}{|c|}{ *Interaction: smoking $\times$ GSTT1-null } & $-111.9(123.1) 0.182$ & $-96.8(122.5) 0.215$ & \\
\hline \multicolumn{6}{|l|}{ GSTM1 } \\
\hline Present & Non-smoking $(n=242)$ & 3413.6 & Referent & Referent & Referent \\
\hline Present & Smoking $(n=49)$ & 3255.9 & $-87.2(73.9) 0.119$ & $-85.1(74.2) 0.126$ & $-58.8(66.1) 0.187$ \\
\hline Null & Non-smoking $(n=214)$ & 3363.9 & Referent & Referent & $-32.4(41.2) 0.216$ \\
\hline Null & Smoking $(n=34)$ & 3324.8 & $-97.1(92.0) 0.146$ & $-100.6(90.2) 0.133$ & $-118.7(79.6) 0.069$ \\
\hline \multicolumn{2}{|c|}{ *Interaction: smoking XGSTM1-null } & & $-17.0(106.1) 0.437$ & $-26.6(105.6) 0.400$ & \\
\hline \multicolumn{6}{|c|}{ GSTT1 \& GSTM1 } \\
\hline Present & Non-smoking $n=207$ ) & 3429.4 & Referent & Referent & Referent \\
\hline Present & Smoking $(n=38)$ & 3251.2 & $-137.9(82.3) 0.048$ & $-135.5(82.6) 0.051$ & $-84.7(71.2) 0.118$ \\
\hline Null & Non-smoking $(n=36)$ & 3339.7 & Referent & Referent & $10.1(76.1) 0.447$ \\
\hline$\overline{\text { Null }}$ & Smoking $(n=11)$ & 3093.5 & $-318.0(198) 0.058$ & $-320.8(203) 0.061$ & $-311.2(128) 0.008$ \\
\hline
\end{tabular}

Interaction: smoking XGSTT1-null XGSTM1-null

$-234.5(164.3) 0.078$

$B$ represent the difference in mean birth weight for cigarette smoking between the variant genotype.

$+B$ crude.

++ $\beta$ after adjustment for the covariates: gestational age, body mass index, education, family status, parity and blood pressure

*Test of interaction: a $\mathrm{P}$ value is presented for testing the null hypothesis, $B=0$ in multiple linear regression models for the product term, smoking $\mathrm{x}$ genotypes. 
Women reporting three or more stressful life events were significantly more likely to have a low birthweight infant after controlling for smoking and other sociodemographic covariates [38].

Findings of this study provide additional data supporting the conclusion that light maternal smoking during pregnancy may lead to reduced birthweight in infants. Our results corroborate the results of other studies that identification of the group of susceptible subjects should be based on both environmental exposure and gene polymorphism and that the individual differences in metabolic activation and detoxification of xenobiotics partly depends on the genetic polymorphisms associated with the GST enzymes [13-15,18,24,34]. When the GSTT1 genotype is considered in smoking pregnant women, the three different studies estimated reduction in birthweight among the GSTT1-plus and GSTT1-null groups was as follows: $43 \mathrm{~g}$ $(\mathrm{P}=0.48)[14], 222 \mathrm{~g}(P<0.05)[15]$, and $642 \mathrm{~g}(P<0.001)$ [13]. When the GSTM1 genotype is considered, the estimated reduction in birthweight between GSTM1-plus and GSTM1-null groups is $171 \mathrm{~g}(P=0.04)$ [14] and $222 \mathrm{~g}$ $(P<0.05)$, respectively [15]. The effects on the reduction of birthweight are not observed among women with GSTM1-null or GSTT1-null genotypes who had never smoked and the data have been adjusted to the main confounding factors.

A combination of the GSTM1-null and the GSTT1null genotypes has been found to exacerbate the effect of maternal exposure to environmental tobacco-smoking on the birthweight of infants more than the presence of either genotype alone. Our previous publicised study have shown that when both GSTM1 and GSTT1 genotypes were considered, the greater increase in low birth weight and intra-uterine growth restriction risk was found among smoking mothers with the GSTM1 genotype absent, OR 3.31 [95\% CI 0.60, 18.4] and OR 2.47 [95\% CI 0.31, 13.1], correspondingly [34].

We can postulate that the significant differences between the publicised studies, which are devoted to the effects of tobacco-smoke exposure on birthweight, could be attributed to the diverse ethnic composition of the populations considered in the studies, resulting in different distributions of the GST allelic frequency and different levels of cigarette-smoke exposure, because dose-response gradients in relation to the number of cigarettes smoked do exist [19]. Furthermore, these results may be affected by the residual uncontrolled confounding variables, such as prepregnancy BMI, hypertension, stress level, gestational age and others, which are negatively or positively associated with birthweight.

The main factors influencing birth-weight reduction are gestational age and the organism's response to toxicity from environmental xenobiotics, such as tobacco PAHs. Tobacco smoke toxins impair placental vasculature function and subsequent transplacental transport of oxygen and nutrients, and may lead to changes in vascular resistance $[39,40]$. Reduction in blood flow increase apoptosis and it is possible that this could be one of the mechanisms playing a role in the growth restriction [41].

Several recent studies have investigating how genetic susceptibility modulates risk of adverse pregnancy outcomes from environmental exposures such as cigarette smoke. Toxic chemicals could disturb foetal and placental cellular regulation via elevated DNA adducts and DNA damage [42]. Oxidative damage to placental DNA and increased levels of 8-oxodG in placental DNA can result in intrauterine growth restriction and low birthweight [43]. Maternal tobacco smoke exposure at an epigenome-wide level is associated with placental gene expression and DNA differential methylation and smoking-mediated birthweight reduction [44].

It is likely that smoking mothers with high-risk genotypes may have higher levels of PAH-DNA adducts and DNA strand breakage due to the increased activity of enzymes that metabolize cigarette toxins (e.g., CYP1A1 Aa and aa) and lower or absent activity of enzymes that detoxify these compounds (e.g. GSTT1-null, GSTM1-null genotypes) [45]. Moreover, such gene-smoking interactions may exert their synergistic effects on birthweight through maternal and foetal inflammatory responses and immune responses [46]. As reported by some authors, maternal exposure to tobacco smoke induces oxidative stress. Furthermore, maternal genetic polymorphisms related to GSTM1 and GSTT1 may modify the oxidative stress caused by maternal exposure to tobacco smoke [47].

We have investigated the genetic effects and the geneenvironment interaction by controlling for major confounding variables. This study has the advantage of being the first to show that even light maternal smoking, in association with double-null GSTT1 and GSTM1 genotypes, might significantly decrease the infant birthweight. In this study, we estimated that the percentage of GSTT1-null genotype was $17.2 \%$ and that of GSTM1 was $46.0 \%$. The carriers of double-null genotypes were $8.7 \%$ of the total population studied.

When the results of this study are interpreted, a few conditions should be considered. This is a low-risk population with low-level tobacco smoke exposure (4.8 cig./ day) and low prevalence of GSTT1-null genotype; these factors may limit the extrapolation of these results to other populations. One of the limitations of the study is the relatively small sample size with the GSTT1-null genotype. The evaluation of exposure to tobacco smoke was indirect; we used self-reported information on smoking during pregnancy, and thus the possibility of bias in both reporting and exposure classification exists. We also examined phase-II metabolic genes without study genes expressed in phase-I. However, in this study, we controlled for the main 
variables that might confound the association between maternal smoking, genetic polymorphism and birthweight; therefore, the residual confounding of the results by smoking is expected to be small. Despite these limitations, findings of this study provide additional data supporting the conclusion that maternal smoking during pregnancy may lead to reduced birth weight in newborns.

\section{Conclusion}

The study shows the modifying effect of the GSTT1 and GSTM1 genotypes on birthweight among smoking women and presents evidence that carriers of the null genotypes should be treated as an increased susceptibility group for infant birthweight decrease. Our findings provide additional insight into the biological determinants of response to environmental exposure based on the combination of genes and individual characteristics. Genotyping for the GSTT1 and GSTM1 polymorphisms, simple and inexpensive assays, could be suitable biomarkers identifying genetically susceptible pregnant women. These risk stratification markers could provide a valuable approach to estimate the "causal" effects of risk behaviours with genetic-predisposing factors (such as smoking) and could lead to targeted smoking cessation interventions during pregnancy as prevention for infants with low birthweight. The GSTT1-null genotype, either presents only one or both with GSTM1-null genotype in a single subject, may have a modifying effect on birthweight among smoking women even though their smoking is low level. Our data also show that identification of a susceptible-subject group should be based on both environmental exposure and gene polymorphism.

\section{Competing interest}

We confirm that all authors have no actual or potential competing interests regarding the submitted article and the nature of those interests.

\section{Authors' contributions \\ $A D$ was involved in primary data collection as well as the coding and analysis of data, and the preparation of the manuscript. RG was involved in the conceptualization of the research, the preparation of the manuscript. AP contributed to genetic analysis. RD contributed to the development of the survey instrument, contributed to data acquisition. MJN contributed to qualitative data analysis and contributed to revisions of the manuscript. All authors read and approved the final manuscript.}

\section{Acknowledgements}

This work was supported in part by grant FP6-036224 from the European Commission. We acknowledge the contribution of the Clinics of Lithuanian University of Health Science involved in registration of pregnancy outcomes.

\section{Funding}

This work was supported in part by grant FP6-036224 from the European Commission

\footnotetext{
Author details

${ }^{1}$ Department of Environmental Sciences, Vytautas Magnus University, Donelaicio st. 58, 44248, Kaunas, Lithuania. ${ }^{2}$ Clinic of Obstetrics and Gynecology, Lithuanian University of Health Science, Kaunas, Lithuania. ${ }^{3}$ Center for Research in Environmental Epidemiology (CREAL), Parc de Recerca Biomedica de Barcelona - PRBB, Barcelona, Spain.
}

Received: 27 June 2012 Accepted: 19 December 2012

Published: 26 December 2012

\section{References}

1. Stillerman KP, Mattison DR, Giudice LC, Woodruff TJ: Environmental exposures and adverse pregnancy outcomes: a review of the science. Reproductive Sci 2008, 15:631-650.

2. Lee BE, Hong YC, Park H, Ha M, Koo BS, Chang N, Roh YM, Kim BN, Kim YJ, Kim BM, Jo SJ, Ha EH: Interaction between GSTM1/GSTT1 polymorphism and blood mercury on birth weight. Environ Health Perspect 2010, 118:437-443.

3. Windham GC, Hopkins B, Fenster L, Swan SH: Prenatal active or passive tobacco smoke exposure and the risk of preterm delivery or low birth weight. Epidemiology 2000, 11:427-433.

4. Matijasevich A, Brion MJ, Menezes AM, Barros AJD, Santos IS, Barros FC: Maternal smoking during pregnancy and offspring growth in childhood: 1993 and 2004 Pelotas cohort studies. Arch Dis Child 2011, 96:519-525.

5. Savitz DA, Dole N, Terry JW Jr, Zhou H, Thorp JM: Smoking and pregnancy outcome among African-American and white women in central North Carolina. Epidemiology 2001, 12:636-642.

6. Bolat F, Eren O, Bolat G, Can E, Cömert S, Uslu HS, Nuhoğlu A: Maternal smoking during pregnancy and eff ects on neonatal anthropometry: a prospective study. Turk J Med Sci 2012, 42:999-1005.

7. Varvarigou AA, Fouzas S, Beratis NG: Effect of prenatal tobacco smoke exposure on fetal growth potential. J Perinat Med 2010, 38:683-687.

8. Pollack H, Lantz PM, Frohna JG: Maternal smoking and adverse birth outcomes among singletons and twins. Am J Public Health 2000, 90:395-400.

9. Zeka A, Melly SJ, Schwartz J: The effects of socioeconomic status and indices of physical environment on reduced birth weight and preterm births in Eastern Massachusets. Environ Health 2008, 7:60.

10. Kukla L, Hruba D, Tyrlik M: Smoking and damages of reproduction: evidence of ELSPAC. European longitudinal study of the pregnancy and childhood. Cent Eur J Public Health 2001, 9:59-63.

11. Zhu BQ, Heeschen C, Sievers RE, Karliner JS, Parmley WW, Glantz SA, Cooke JP: Second hand smoke stimulates tumor angiogenesis and growth. Cancer Cell 2003, 4:191-196.

12. Sasaki S, Sata F, Katoh S, Saijo Y, Nakajima S, Washino N, Konishi K, Ban S, Ishizuka M, Kishi R: Adverse birth outcomes associated with maternal smoking and polymorphisms in the $n$-nitrosamine-metabolizing enzyme genes NQO1 and CYP2E1. Am J Epidemiol 2008, 167:719-726.

13. Wang X, Zuckerman B, Pearson C, Kaufman G, Chen C, Wang G, Niu T, Wise $\mathrm{PH}$, Bauchner $\mathrm{H}, \mathrm{Xu}$ X: Maternal cigarette smoking metabolic gene polymorphism and infant birth weight. JAMA 2002, 287:195-202.

14. Sasaki S, Kondo T, Sata F, Saijo Y, Katoh S, Nakajima S, Ishizuka M, Fujita S, Kishi R: Maternal smoking during pregnancy and genetic polymorphisms in the Ah receptor, CYP1A1 and GSTM1 affect infant birth size in Japanese subjects. Mol Hum Reprod 2006, 12:77-83.

15. Sram RJ, Binkova B, Dejmek J, Chvatalova I, Solansky I, Topinka J: Association of DNA adducts and genotypes with birth weight. Mutat Res 2006, 608:121-128.

16. Infante-Rivard C, Weinberg CR, Guiguet M: Xenobiotic-metabolizing genes and small-for-gestational-age births: interaction with maternal smoking. Epidemiology 2006, 17:38-46.

17. Seidegard J, Ekstrom G: The role of human glutathione transferases and epoxide hydrolases in the metabolisms of xenobiotics. Environ Health Perspect 1997, 105:791-799.

18. Hong YC, Lee KH, Son BK, Ha EH, Moon HS, Ha M: Effects of the GSTM1 and GSTT1 polymorphisms on the relationship between maternal exposure to environmental tobacco smoke and neonatal birth weight. J Occup Environ Med 2003, 45:492-498.

19. Nukui T, Day RD, Sims CS, Ness RB, Romkes M: Maternal/newborn GSTT1 null genotype contributes to risk of preterm, low birthweight infants. Pharmacogenetics 2004, 14:569-576.

20. Hayes JD, Strange RC: Glutathione S-transferase polymorphisms and their biological consequences. Pharmacology 2000, 61:154-166.

21. Peter H, Deutschmann S, Reichel D, Hallier E: Metabolism of methyl chloride by human erythrocytes. Arch Toxicol 1989, 63:351-355.

22. Warholm M, Rane A, Alexandrie AK, Monaghan G, Rannug A: Genotypic and phenotypic determination of polymorphic glutathione transferase T1 in a Swedish population. Pharmacogenetics 1995, 5:252-254. 
23. Jain M, Kumar S, Rastogi N, Lal P, Ghoshal UC, Tiwari A, Pant MC, Baiq MQ, Mittal B: GSTT1, GSTM1 and GSTP1 genetic polymorphisms and interaction with tobacco, alcohol and occupational exposure in esophageal cancer patients from North India. Cancer Lett 2006, 242:60-67.

24. Kishi R, Sata F, Yoshioka E, Ban S, Sasaki S, Konishi K, Washino N: Exploiting gene-environment interaction to detect adverse health effects of environmental chemicals on the next generation. Basic Clin Pharmacol Toxicol 2008, 102:191-203.

25. Delpisheh A, Brabin L, Topping J, Reyad M, Tang AW, Brabin BJ: A case-control study of CYP1A1, GSTT1 and GSTM1 gene polymorphisms, pregnancy smoking and fetal growth restriction. Eur J Obstet Gynecol Reprod Biol 2009, 143:38-42.

26. Chen X, Abdulhamid I, Woodcroft KJ: Maternal smoking during pregnancy, polymorphic CYP1A1 and GSTM1, and lung-function measures in urban family children. Environ Res 2011, 11:1215-1221.

27. Breton CV, Salam MT, Vora H, Gauderman WJ, Gilliland FD: Genetic variation in the glutathione synthesis pathway, air pollution, and children's lung function growth. Am J Respir Crit Care Med 2011, 183:243-248.

28. Gilliland FD, Li YF, Dubeau L, Berhane K, Avol E, McConnell R, Gauderman WJ, Peters JM: Effects of glutathione S-transferase M1, maternal smoking during pregnancy, and environmental tobacco smoke on asthma and wheezing in children. Am J Respir Crit Care Med 2002, 166:457-463.

29. Infante-Rivard C, Amre D, Sinnett D: GSTT1 and CYP2E1 polymorphisms and trihalomethanes in drinking water: effect on childhood leukemia. Environ Health Perspect 2002, 110:591-593.

30. Jurado D, Munoz C, Luna Jde D, Munoz-Hoyos A: Is maternal smoking more determinant than paternal smoking on the respiratory symptoms of young children? Respir Med 2005, 99:1138-1144.

31. Whyatt RM, Perera FP, Jedrychowski W, Santella RM, Garte S, Bell DA: Association between polycyclic aromatic hydrocarbon-DNA adduct levels in maternal and newborn white blood cells and glutathione S-transfer- ase P1 and CYP1A1 polymorphisms. Cancer Epidemiol Biomarkers Prev 2000, 9:207-212.

32. Dejmek J, Solansky I, Benes I, Lenícek J, Srám RJ: The impact of polycyclic aromatic hydrocarbons and fine particles on pregnancy outcome. Environ Health Perspect 2000, 108:1159-1164.

33. Chen X, Woodcroft KJ: Polymorphisms in metabolic genes CYP1A1 and GSTM1 and changes in maternal smoking during pregnancy. Nicotine Tob Res 2009, 11:225-233.

34. Grazuleviciene R, Danileviciute A, Nadisauskiene R, Vencloviene J: Maternal Smoking, GSTM1 and GSTT1 polymorphism and susceptibility to adverse pregnancy outcomes. Int J Environ Res Public Health 2009, 6:1282-1297.

35. Arand M, Mühlbauer R, Hengstler J, Jäger E, Fuchs J, Winkler L, Oesch F: A multiplex polymerase chain reaction protocol for the simultaneous analysis of the glutathione S-transferase GSTM1 and GSTT1 polymorphisms. Anal Biochem 1996, 236:184-186.

36. Hruba D, Kachlik P: Influence of maternal active and passive smoking during pregnancy on birthweight in newborns. Cent Eur J Public Health 2000, 8:249-252.

37. Oberg S, Ge D, Cnattingius S, Svensson A, Treiber FA, Snieder H, lliadou A: Ethnic differences in the association of birth weight and blood pressure. Am J Hypertens 2007, 20:1235-1241.

38. Brown SJ, Yelland JS, Sutherland GA, Baghurst PA, Robinson JS: Stressful life events, social health issues and low birthweight in an Australian population-based birth cohort: challenges and opportunities in antenatal care. BMC Public Health 2011, 11:196.

39. Gandley RE, Jeyabalan A, Desai K, McGonigal S, Rohland J, DeLoia JA Cigarette exposure induces changes in maternal vascular function in a pregnant mouse model. Am J Physiol Regul Integr Comp Physiol 2010, 298:R1249-R1256.

40. Machado Jde B, Plínio Filho VM, Petersen GO, Chatkin JM: Quantitative effects of tobacco smoking exposure on the maternal-fetal circulation. BMC Pregnancy Childbirth 2011, 31:11-24.

41. Voght Isaksen C: Maternal smoking, intrauterine growth restriction, and placental apoptosis. Pediatr Dev Pathol 2004, 7:433-442.

42. Tsai HJ, Liu X, Mestan K, Yu Y, Zhang S, Fang Y, Pearson C, Ortiz K, Zuckerman B, Bauchner H, Cerda S, Stubblefield PG, Xu X, Wang X: Maternal cigarette smoking, metabolic gene polymorphisms, and preterm delivery: new insights on GxE interactions and pathogenic pathways. Hum Genet 2008, 123:359-369.
43. Rossner PJ, Tabashidze N, Dostal M, Novakova Z, Chvatalova I, Spatova M, Sram RJ: Genetic, biochemical, and environmental factors associated with pregnancy outcomes in newborns from the Czech Republic. Environ Health Perspect 2011, 119:265-271.

44. Suter M, Ma J, Harris AS, Patterson L, Brown KA, Shope C, Showalter L, Abramovici A, Aagaard-Tillery KM: Maternal tobacco use modestly alters correlated epigenome-wide placental DNA methylation and gene expression. Epigenetics 2011, 6:1284-1294

45. Perera FP, Rauh V, Whyatt RM, Tsai WY, Bernert JT, Tu YH, Andrews H, Ramirez J, Qu L, Tang D: Molecular evidence of an interaction between prenatal environmental exposures and birth outcomes in a multiethnic population. Environ Health Perspect 2004, 112:626-630.

46. Noakes PS, Holt PG, Prescott SL: Maternal smoking in pregnancy alters neonatal cytokine responses. Allergy 2003, 58:1053-1058.

47. Park EY, Hong YC, Lee KH, Im MW, Ha E, Kim YJ, Ha M: Maternal exposure to environmental tobacco smoke, GSTM1/T1 polymorphism and oxidative stress. Reprod Toxicol 2008, 26:197-202.

doi:10.1186/1471-2393-12-161

Cite this article as: Danileviciute et al:: Low level maternal smoking and infant birthweight reduction: genetic contributions of GSTT1 and GSTM1 polymorphisms. BMC Pregnancy and Childbirth 2012 12:161.

\section{Submit your next manuscript to BioMed Central and take full advantage of:}

- Convenient online submission

- Thorough peer review

- No space constraints or color figure charges

- Immediate publication on acceptance

- Inclusion in PubMed, CAS, Scopus and Google Scholar

- Research which is freely available for redistribution 